\title{
Characterization of Postmortem Biochemical Changes in Rabbit Plasma Using ATR-FTIR Combined With Chemometrics: A Preliminary study
}

Ji Zhang \& Bing Li \& , Qi Wang, Chengzhi Li, Yinming Zhang, Hancheng Lin, Zhenyuan Wang ${ }^{*}$

\begin{abstract}
Postmortem interval (PMI) determination is one of the most challenging tasks in forensic medicine due to a lack of accurate and reliable methods. It is especially difficult for late PMI determination. Although many attempts with various types of body fluids based on chemical methods have been made to solve this problem, few investigations are focused on blood samples. In this study, we employed an attenuated total reflection (ATR)-Fourier transform infrared (FTIR) technique coupled with principle component analysis (PCA) to monitor biochemical changes in rabbit plasma with increasing PMI. Partial least square (PLS) model was used based on the spectral data for PMI prediction in an independent sample set. Our results revealed that postmortem chemical changes in compositions of the plasma were time-dependent, and various components including proteins, lipids and nucleic acids contributed to the discrimination of the samples at different time points. A satisfactory prediction within $48 \mathrm{~h}$ postmortem was performed by the combined PLS model with a good fitting between actual and predicted PMI of 0.984 and with an error of $\pm 1.92 \mathrm{~h}$. In consideration of the simplicity and portability of ATR-FTIR, our preliminary study provides an experimental and theoretical basis for application of this technique in forensic practice.
\end{abstract}

Key words: postmortem interval, plasma, Fourier transform infrared spectroscopy, partial least 
square, principle component analysis

\section{Introduction}

Determination of a postmortem interval (PMI), the time period between death and examination of the deceased, is of great importance in forensic medicine, especially in homicide or unwitnessed investigations[1]. PMI determination is helpful for narrowing the search of suspects based on their locations at the time of death and reconstructing a crime scene. However, traditional approaches that are largely based on postmortem changes in the corpse, such as algor mortis, livor mortis, distribution of rigor mortis and turbidity of the cornea, are still unable to perform an accurate and reliable PMI estimation[2] because they either solely depend on subjective observations that can be misleading or are difficult to make an assessment during the late phase[3] Thus, as one of the constant and central topics in forensic research, development of objective and reproducible methods for PMI estimation is urgently needed, especially for late postmortem stages $(>24 h)$.

Numerous chemical techniques have been proposed in characterization of postmortem changes in biological tissues, leading to the establishment of the field called "thanatochemistry"[4]. In this field, many efforts are directed towards biochemical examination of different body fluids such as vitreous humor (VH), cerebrospinal fluid (CSF), pericardial fluid and synovial fluid [5-8] because they are readily available at a crime scene or during autopsy. However, few studies on PMI estimation are based on blood sample although it is commonly used in forensic toxicology. Earlier studies showed that there was a linear relationship of high correlation between a few parameters in blood samples and PMI $[9,10]$. Afterwards, with the development of more advanced biochemistry techniques, more and more substances in the blood samples at different postmortem time points can be monitored in vivo and in vitro [11] . Notably, Takako and his colleagues recently construct a PMI prediction model based on multiple metabolic products in rat plasma detected by a GC-MS/MS-based metabolic method. The model is validated by an independent sample set and obtains a satisfactory prediction result[12], which suggests that PMI estimation methods based on broader chemical components may be more powerful than those using only single or a few parameters. Taken together, previous studies support the use of blood 
sample (including whole blood, serum and plasma) as a promising candidate for PMI delimitation.

Attenuated total reflectance-Fourier transform infrared (ATR-FTIR) technique is a valuable bio-analytic tool according to vibrational motions of functional molecular groups. It has been widely applied in biomedical and life science due to its many advantages in the study of biological specimens. In contrast to other analytic techniques, ATR-FTIR can offer rapid, cost-effective and high-quality chemical analysis with simple or no need of sample preparation[13, 14]. It is already known that biological tissues contain a variety of macromolecules including protein, lipid, nucleic acid and carbohydrate, all of which have particular absorption bands in the infrared frequency domain. Therefore, the ability of ATR-FTIR to simultaneously probe multiple compositions and structures of biomolecules provides an ideal platform for biochemical investigations. Meanwhile, the utility of chemometrics enables the technique to identify subtle spectral differences associated with various physiological and pathological processes and then converts that information into a predicated classifier or discriminator. Currently, there are several papers reporting the investigations of different physiological and disease states in blood components using ATR-FTIR coupled with chemometrics [15-18].

Our work is focused on the characterization of postmortem changes in biological tissues with the ATR-FTIR technique in order to determine the time after death. In our previous studies, it has been demonstrated that various absorption intensities or area ratios in an infrared spectrum are strongly correlated with PMI in human and animal tissues including brain, kidney cortex, cardiac muscle and lung [19-23]. However, these studies did not make full use of ATR-FTIR for PMI delimitation because only a few parameters were taken into consideration.

In this study, we utilized ATR-FTIR to interrogate rabbit plasma harvested at different time points within $48 \mathrm{~h}$ postmortem. The obtained spectral datasets were analyzed using principle component analysis (PCA) in order to visualize classification of different postmortem time points and determine distinguishing spectral features. Partial least square (PLS) classification models based on the resulting spectral dataset were then built and validated by an independent sample set which was not included in the modeling dataset.

\section{Materials and methods}

\subsection{Animal experiments and sample preparation}


The study was conducted in strict accordance with the recommendations in the Guide for the

87 Care and Use of Laboratory Animals of Xian Jiaotong University. The protocol was approved by 88 the Committee on the Ethics of Animal Experiments of Xian Jiaotong University. All surgery was performed under sodium pentobarbital anesthesia, and all efforts were made to minimize animal suffering. A total of 105 male Japanese white rabbits weighing 2-2.5 kg were bought from Xian Laboratory Animal Research Center (Xian, China). All animals were acclimatized for three days before experiments and were exposed to a $12 \mathrm{~h}$-light-dark cycle with free access to water and standard diet. The rabbits were sacrificed by injection of the air into the auricular vein after anesthesia. The corpses were then placed in an environmental chamber at a constant temperature of $25{ }^{\circ} \mathrm{C}$ and relative humidity of $40 \%$. Blood samples were collected from the right ventricle of the heart, and plasma was rapidly harvested by centrifugation at $14000 \mathrm{rpm}$ for $15 \mathrm{~min}$. Plasma samples were then quickly frozen in liquid nitrogen until FTIR analysis.

All rabbits were divided into calibration and validation groups. In calibration group, the plasma samples from 81 rabbits were collected at $0 \mathrm{~h}, 6 \mathrm{~h}, 12 \mathrm{~h}, 18 \mathrm{~h}, 24 \mathrm{~h}, 30 \mathrm{~h}, 36 \mathrm{~h}, 42 \mathrm{~h}$ and $48 \mathrm{~h}$ postmortem respectively (9 rabbits per time point), whose spectral variables were used to establish PLS models and for chemical analysis of PCA. Moreover, it was essential to create the validation group, whose PMI were not included in the calibration group, for assessment of the developed PLS model. Therefore, the plasma samples of the remaining 24 rabbits were additionally collected from $3 \mathrm{~h}$ to $45 \mathrm{~h}$ at a $6 \mathrm{~h}$ interval ( 3 rabbits per time point).

\subsection{ATR-FTIR spectroscopic measurement and quality control}

After thawing on an ice bath, approximately $100 \mu \mathrm{l}$ plasma for each animal was shaken by a vortex mixer, and then mixed repeatedly using a micropipettor before FTIR measurement. $1 \mu \mathrm{L}$ of each sample was deposited exactly on a $250 \mu$ m-diameter ATR diamond window (Smart Orbit Diamond ATR, Thermo Fisher, USA).The drops were placed naturally in a temperature-controlled laboratory $\left(37^{\circ} \mathrm{C}\right)$ for $20 \mathrm{~min}$, and then dried sufficiently by an air dryer for about 30s. This procedure was completed by the same analyst. The spectra were obtained within the frequency range of $4000-900 \mathrm{~cm}^{-1}$ at a resolution of $4 \mathrm{~cm}^{-1}, 32$ scans and 2 zero filling factors using a Nicolet 5700 FTIR spectrometer (Thermo Scientific Nicolet, CA). The background spectra collected on the clear ATR window under the same acquisition condition were automatically subtracted from 
the spectra of each sample. To get rid of atmospheric water vapour, we kept the indoor relative humidity under $20 \%$ using a dehumidifier. The spectra from 9 independent aliquots of one sample were recorded to check its reproducibility and to evaluate possible errors resulting from the instrument's working state and manual sample loading. It took about 30s for the collection of each spectrum. Moreover, a quality test was run to remove raw spectra that had poor signal-noise ratio and maximum absorbance value outside the range of 0.3-0.5. If the spectra do not pass the test, repeated spectra from the same sample were obtained under the same acquisition mode. Following this, 9 replicates were averaged to produce a spectrum for one animal, and this procedure was repeated for all animals. Moreover, the standard deviations of the spectra from the same sample and PMI group were also calculated for assessment of within-sample and within-group variations.

\subsection{Data pre-processing}

The raw absorbance spectra which had passed the quality test were further pre-processed by Matlab R2014a (MathWorks, U.S.A) equipped with PLS Toolbox 8.1.1 (Eigenvector Research, Inc.). The raw spectrum was converted into a second derivative with Savitzkmxy-Golay algorithm (23 smoothing points) and then normalized by the extended multiplicative signal correction (EMSC) for resolving overlapped peaks and correcting baseline offset. The second derivative FTIR spectra before and after the EMSC correction are shown in Fig. S1. The interference region from the diamond ATR crystal $\left(1790-2750 \mathrm{~cm}^{-1}\right)$ was excluded for all the spectra because this region does not contain any characteristic peaks of blood[24, 25].

\subsection{Multivariable statistical analysis}

The PCA and PLS algorithm based on the processed second derivative spectra within the bio-region from 1800-900 and 3100-2800 $\mathrm{cm}^{-1}$ were also established by Matlab R2014a (MathWorks, U.S.A) equipped with PLS Toolbox 8.1.1 (Eigenvector Research, Inc.).

PCA was employed to explain the covariance structure of data by using a small number of components which is a linear combination of original variables[26]. Specifically, PCA extracts a set of correlated variables, and then transforms them into a smaller set of uncorrelated variables referred to as principle component (PC), while maintaining as much of the information in the original data as possible. The classification information is expressed by the projection of the samples on a set of orthogonal PCs, and the contribution of variables into it can be evaluated by loading plots associated with corresponding PCs. In this study, leave-one-out cross-validation (CV) 
was performed, and eight PCs were selected for initial PCA decomposition. The two dimensional score plot along PC1 versus PC2 and corresponding loading plots were graphed in order to identify the potential spectral features for PMI estimation.

The model of simple partial least squares (PLS 1) was established for PMI prediction. Unlike PCA, this supervised model can extract simultaneously components (referred to as latent factors) from the predictor variables $\mathrm{X}$ and the response variables $\mathrm{Y}$ for constructing a predictive model. In this study, the predictor variables $\mathrm{X}$ corresponded to the matrix of spectral intensity while the response variables Y were associated with PMI values. As reported previously, the performance of the models were evaluated based on two validation procedures including internal and external validations [27, 28]. For the internal validation procedure, leave-one-out cross-validation (CV) were performed using the calibration dataset through the root mean square error of CV (RMSECV) and $\mathrm{R}^{2}$. In the external validation, the developed models were assessed using the validation dataset that were not included in the calibration process through $\mathrm{Q}^{2}$ and the root mean square error of predication (RMSEP). $\mathrm{R}^{2}$ and $\mathrm{Q}^{2}$ explained the goodness of fitting between actual and predicted values in the model, and the value above 0.9 was considered very good. The optimized number of latent factors was determined when the minimum value of $\mathrm{R}^{2}$ was reached. RMSECV and RMSEP generally represented the global model error and should be as small as possible. The regression loading plots were graphed as a function of spectral frequency in order to visualize the importance of the spectral variables to the model.

\section{Results and discussion}

In order to enhance spectral reproducibility and quality, the spectra from one sample were collected by only one analyst under the same laboratory conditions. Moreover, it is essential to perform a quality test for restricting within-sample and within-group spectral variations. Notably, our pre-experiment found that poor signal-noise ratio, Mie-scattering and variability of replicated spectra became more evident when the maximum absorbance in one spectrum was below 0.3 . Therefore, the lower limit was set to this value. An example of similar spectra from a sample was illustrated in Fig.S2, where comparable 9 replicated spectra were observed with minor standard deviation and were not affected by the resonance Mie scattering and water vapour. The mean spectra with their standard deviations in all PMI groups were shown in Fig.S3, where the 
maximum absorbance differences within the group was close to 0.05 , which was far below the error margin of the quality test.

A comparison of the average second derivatives of FTIR spectra for the plasma of all PMI groups was presented in Fig 1, where the negative peaks were directly aligned to the center of the absorbance peaks of the non-derivative spectrum. The assignment of the main absorption bands was summarized in Table 1. The spectra contained several bands due to vibrations of different functional groups of biomolecules such as protein, lipid, carbohydrate and nucleic acid. This demonstrates that different biological components in the plasma can be discriminated by the ATR-FTIR experimental technique. Furthermore, it is clearly seen from the figure that all FTIR spectra appear to overlap in the frequency region of $1800-900 \mathrm{~cm}^{-1}$ and $3100-2800 \mathrm{~cm}^{-1}$ with fluctuations in some peak intensities among PMI groups. This finding suggests that it is nearly impossible by visualization of intensity changes in a single or a few absorption peaks to distinguish PMI groups due to the complexity and heterogeneity of postmortem changes. For a clear and objective assessment of spectral variance across the studied PMI groups we employed multivariate data analysis by PCA, whose scores and loading plots reveal subtle changes appearing in the plasma over the postmortem time.

\subsection{Characterization of biochemical changes in the plasma by PCA}

In our study, PCA scores plot (see in Fig. 2A) was obtained along PC 1 versus PC 2 because the two PCs explained most variances (94.5\%) of the original data and showed a desirable classification between the PMI groups. Fig. 2A illustrates that spectral points are clustered based on their PMI groups and positioned from left to right with increasing postmortem time points along PC1. This demonstrates that postmortem compositions in the plasma vary significantly between time points, and the biochemical changes are time-dependent. Due to the distribution of PMI groups along PC 1, its loading plot demonstrates spectral features which contribute greatly to the discrimination of plasma samples at different postmortem time points (see in Fig 2B). In Fig. $2 \mathrm{~B}$, the loadings from positive to negative values appear to indicate a general evolution of plasma compositions with development of postmortem changes. The largest alterations were associated with proteins within $1700-1500 \mathrm{~cm}^{-1}$. The discriminating positively correlated loadings were found for the antemortem group at $1633 \mathrm{~cm}^{-1}$ and $1515 \mathrm{~cm}^{-1}$ (corresponding to $\mathrm{C}=\mathrm{O}$ vibrations of $\beta$-sheet of proteins and C-C vibration of tyrosine, respectively) [29-31] whereas negative loadings, 
characteristic for alterations with increasing PMI, include $1650 \mathrm{~cm}^{-1}$ and $1547 \mathrm{~cm}^{-1}$, both of which represent $\alpha$-helical structures [29-32]. This suggests that the proteins rich in $\alpha$-helix structures become predominant in the plasma as the postmortem time progresses. This finding is likely to be associated with hemoglobin impregnation into the plasma because hemoglobin is charactrised by $\alpha$-helical content with a prominent absorption peak around $1650 \mathrm{~cm}^{-1}[33,34]$. However, there have been no studies which confirm whether it is meaningful for PMI determination. Moreover, we also cannot exclude the possibility that other proteins with similar secondary structures would be responsible for these spectral features. Indeed, numerous studies have proposed that some specific proteins or their degradation products in multiple biological specimens may serve as biomarkers for PMI estimation.[35-38]. Nevertheless, there is so far a lack of parallel investigations on animal or human blood specimens. Therefore, our FTIR analysis demonstrates that some proteins with predominant $\alpha$-helix structures could be associated with differences of plasma compositions at different postmortem time points.

Our study also reveals a shift of ester $\mathrm{C}=\mathrm{O}$ stretching motions from 1744 to $1715 \mathrm{~cm}^{-1}$. Since it is found that the band around $1709 \mathrm{~cm}^{-1}$ originates from $\mathrm{C}=\mathrm{O}$ stretch vibration of fatty acids[18], this shift suggests the occurrence of lipid decomposition in the plasma. Interestingly, this finding was not supported by the presence of distinctly negative loadings at $1564 \mathrm{~cm}^{-1}$ and $1399 \mathrm{~cm}^{-1}$. Both the absorption bands have been assigned to asymmetric and symmetric vibrations of $\mathrm{COO}^{-}$ for free fatty acids previously $[31,39]$. However, some studies point out that infrared $\mathrm{COO}^{-}$ stretches also correlate with coordination of proteins to metal ions such as $\mathrm{Ca}^{2+}$ and $\mathrm{Mg}^{2+}[40,41]$.

224 Therefore, the observed strong positive loadings related to $\mathrm{COO}^{-}$stretching vibrations perhaps imply diminishing interaction of plasma proteins with metal ions, which reflects functional and structural changes of certain enzymes in the postmortem plasma.

The PC1 loading plot shows that the discriminating spectral variables in the high-wavenumber region were also associated with lipid degradation because they primarily originate from $\mathrm{C}-\mathrm{H}$ stretches in the hydrocarbon chains of lipid. The loading profiles within this region were characterised by positive loading for $\mathrm{CH}_{2}$ content (at $2925 \mathrm{~cm}^{-1}$ and $2852 \mathrm{~cm}^{-1}$ ) and negative for

$231 \mathrm{CH}_{3}$ content (at $2864 \mathrm{~cm}^{-1}$ and $2955 \mathrm{~cm}^{-1}$ ) [31, 32]. Few studies investigate the relationship between lipids in the plasma and PMI as a result of their substantial fluctuation over time.

233 Nevertheless, it has been shown that there is a significant postmortem decrease in triglyceride 
concentration up to $72 \mathrm{~h}$ after death which is attributable to hydrolysis by lipoprotein lipase but cholesterol concentration has some fluctuations[42]. In short, the weak lipid-associated loadings suggest that the lipid alterations in the plasma may be related with PMI to some extent.

237 The other wavenumbers responsible for the separation were positively correlated with loadings 238 at 1237, 1115, 1080, 1029, 983 and $926 \mathrm{~cm}^{-1}$ respectively. Since these bands are generally specific 239 for C-O and P-O vibrations of carbohydrates and nucleic acids [29, 31, 32, 39, 43], our results may be related to degradation of both the macromolecules over the postmortem time. It has been reported that DNA fragmentation is a very valuable parameter for PMI assessment. This process continues at a constant rate for 3 days if the cadavers are unaffected by external factors[44-46]. Additionally, evidence shows that a longer PMI has widely varying effects on gene transcription with a generally decreasing trend, and many forensic laboratories have monitored RNA degradation to estimate PMI $[3,47,48]$. The alteration may be reflected here by a detectable positive loading at $1115 \mathrm{~cm}^{-1}$, typical for C-O vibration of RNA[18] . With regard to carbohydrates, glycemia is recognized to be unsuitable as a biochemical parameter for PMI estimation in the forensic community because its blood concentration has unpredictable fluctuations after death[49]. Therefore, other types of carbohydrates or their metabolic products would be responsible for the observed loading pattern below $1300 \mathrm{~cm}^{-1}$ frequency region.

Our study reveals that the molecular vibrations from multiple biological components were correlated with the separation of plasma samples collected at different PMIs. However, it should be emphasized that signal intensity in a second derivative spectrum is mainly caused by curvature of the absorbance spectrum rather than concentration of a certain component. Therefore, it is still necessary to quantify relevant concentrations in the plasma in the further investigation after FTIR analysis based on the second derivative spectra.

\subsection{PMI delimitation by PLS model}

As presented in the PCA results, postmortem changes of multiple substances in the plasma that are involved in PMI distinction can be detected in a FTIR spectrum with the range of 1800-900 and $3100-2800 \mathrm{~cm}^{-1}$. Therefore, the next step of this study was to develop a PLS model for accurate PMI estimation based on that spectral information. Fig 3A illustrates the CV results for the model with $0-48 \mathrm{~h}$ which had a good predicted ability as reflected by $\mathrm{R}^{2}$ above 0.9 , but with a relatively high error (RMSECV: $\pm 2.53 \mathrm{~h}$ ). Furthermore, it seemed that spectral points at $24 \mathrm{~h}$ 
onwards can be differentiable from those in the range from 30 to $48 \mathrm{~h}$, indicating a significant component variance of the plasma between the two time periods. Therefore, considering the influence of the variation in the postmortem process on the model precision, we built two individual PLS models for PMI from 0-24h and 24-48h respectively. In Fig 4 A-B, it is expected that these two models have better performance with lower RMSECV $( \pm 1.27 \mathrm{~h}$ for $0-24 \mathrm{~h} ; \pm 1.84 \mathrm{~h}$ for 24-48h).

To obtain information on which wavenumbers have the most influence on each PLS model, regression loading of three models ( including $0-48 \mathrm{~h}, 0-24 \mathrm{~h}$ and $24-48 \mathrm{~h}$ ) for factor 1 against wavenumber were plotted in Fig 3B and Fig 4C-D respectively. The positive and negative bands in this plot are important in generating linearity in the model. In our study, the patterns of regression loadings for the three models are almost similar to the major bands observed in PCA loading plots, and thus the linearity of the model primarily comes from real spectral changes rather than artifacts such as baseline modulations or noise.

Despite the use of $\mathrm{CV}$ in the calibration, the challenge of the model is the accurate identification of unknown spectral data due to high intra-species biodiversity and the limited size of training/calibration samples. Meanwhile, considering forensic practicality for PMI estimation, it is impossible to include spectral data from all postmortem time points in PLS modeling. Thus, a crucial step after model calibration is an external validation with an independent dataset of spectra whose time points are not included for calibration. Furthermore, in order to make more precise estimations, the prediction strategy was based on the following steps: firstly, the PLS model for 0-48h was applied for separating samples collected before and after $24 \mathrm{~h}$ postmortem; secondly, further PMI estimation was performed by corresponding sub-models for 0-24h and 24-48h based on the first approximate evaluation. The current study shows that the model for $0-48 \mathrm{~h}$ achieved a complete segregation of samples obtained before $24 \mathrm{~h}$ postmortem from the remaining ones as shown in Fig 5A. Fig 5B shows the relationship between actual and predicted PMI by both the sub-models, and a satisfactory prediction, except for $27 \mathrm{~h}$, was obtained with $\mathrm{Q}^{2}$ and RMSEP values of 0.984 and $\pm 1.92 \mathrm{~h}$ respectively.

Based on the data from previous investigations, a comparison of the present method with other reported methods was summarized in Table 2. In our work, the predictive error in the validation dataset is quite lower than those of most reported methods. However, this doesn't mean that the 
FTIR technique is considered more efficient due to different sample species. More importantly, some postmortem factors (i.e. temperature and humidity) which could affect the process of global chemical changes was not taken into account in the current study. Therefore, further work would be primarily focused on evaluating influence of these factors on PMI estimation, especially to human samples.

\section{Conclusions}

To the best of our knowledge, this is the first study demonstrating that FTIR vibrational spectroscopy can be employed as an easy and rapid method for assessment of biochemical changes in rabbit plasma with increasing PMI. Multivariate data analysis may prove to be useful to identify the subtle spectral changes as postmortem changes progress. The finding of this work is helpful to better understand postmortem biochemical changes, which has great significance to evaluate the quality of biological samples for forensic toxicology and biochemical test. Nevertheless, it is obvious that matching experiments on biochemistry and molecular biology are still required in order to further determine the origin of these spectral profiles in the postmortem plasma.

Subsequently, we attempted to establish PLS models based on spectral information obtained here for PMI estimation, and the results showed a satisfactory prediction validated by an independent sample data on the whole. It has been reported that portable ATR-FTIR instruments have been commercially available which yield spectral knowledge almost similar to those obtained from the benchtop instruments [55]. This demonstrates the potential of the spectroscopic method proposed here as a complementary tool to determine PMI in situ with biofluids collected at a crime scene. Therefore, our preliminary study as a starting point provides an experimental and theoretical basis for future practical research.

\section{Acknowledgments}

The research is funded by the National Science Foundation of China (No. 81273335, 81471819) and China Postdoctoral Science Foundation Grant (No. 2016M592801).

\section{References}

[1] M.M. Abo El-Noor, N.M. Elhosary, N.F. Khedr, K.I. El-Desouky, Estimation of Early Postmortem 

Path, 37 (2016) 40-46.

325 [2] T. Sun, T. Yang, H. Zhang, L. Zhuo, L. Liu, Interpolation function estimates post mortem interval under ambient temperature correlating with blood ATP level, Forensic Sci Int, 238 (2014) 47-52.

327 [3] Y.H. Lv, K.J. Ma, H. Zhang, M. He, P. Zhang, Y.W. Shen, N. Jiang, D. Ma, L. Chen, A time course study demonstrating mRNA, microRNA, 18S rRNA, and U6 snRNA changes to estimate PMI in deceased rat's spleen, J Forensic Sci, 59 (2014) 1286-1294.

[4] B. Madea, Is there recent progress in the estimation of the postmortem interval by means of thanatochemistry?, Forensic Sci Int, 151 (2005) 139-149. of post-mortem interval: A comparison between cerebrospinal fluid and vitreous humour chemistry, $\mathrm{J}$

334 Forensic Leg Med, 36 (2015) 144-148.

[6] B. Zilg, S. Bernard, K. Alkass, S. Berg, H. Druid, A new model for the estimation of time of death from vitreous potassium levels corrected for age and temperature, Forensic Sci Int, 254 (2015) $158-166$.

[7] N.K. Tumram, R.V. Bardale, A.P. Dongre, Postmortem analysis of synovial fluid and vitreous humour for determination of death interval: A comparative study, Forensic Sci Int, 204 (2011) 186-190.

[8] C. Palmiere, P. Mangin, Urea nitrogen, creatinine, and uric acid levels in postmortem serum,

341 vitreous humor, and pericardial fluid, Int J Legal Med, 129 (2015) 301-305.

[9] Q. D, Double logarithmic, linear relationship between plasma sodium/potassium concentration ratio

343 and postmortem interval during the 6-96-h postmortem period in rats, Forensic Sci Int. Genetics, 44 344 (1990) 125-134.

345 [10] Q. D, Linearization of the relationship between postmortem plasma chloride concentration and 346 postmortem interval in rats, Forensic Sci Int-Gen, 45 (1990) 117-128.

347 [11] A.E. Donaldson, I.L. Lamont, Biochemistry changes that occur after death: potential markers for 348 determining post-mortem interval, PloS one, 8 (2013) e82011.

349 [12] T. Sato, K. Zaitsu, K. Tsuboi, M. Nomura, M. Kusano, N. Shima, S. Abe, A. Ishii, H. Tsuchihashi, 350 K. Suzuki, A preliminary study on postmortem interval estimation of suffocated rats by 351 GC-MS/MS-based plasma metabolic profiling, Anal Bioanal Chem, 407 (2015) 3659-3665.

352 [13] C. Vigano, L. Manciu, F. Buyse, E. Goormaghtigh, J.M. Ruysschaert, Attenuated total reflection 
IR spectroscopy as a tool to investigate the structure, orientation and tertiary structure changes in peptides and membrane proteins, Biopolymers, 55 (2000) 373-380.

[14] S.G. Kazarian, K.L. Chan, Applications of ATR-FTIR spectroscopic imaging to biomedical samples, BBA, 1758 (2006) 858-867.

[15] K. Gajjar, J. Trevisan, G. Owens, P.J. Keating, N.J. Wood, H.F. Stringfellow, P.L. Martin-Hirsch, F.L. Martin, Fourier-transform infrared spectroscopy coupled with a classification machine for the analysis of blood plasma or serum: a novel diagnostic approach for ovarian cancer, Analyst, 138 (2013) 3917-3926.

[16] J.R. Hands, P. Abel, K. Ashton, T. Dawson, C. Davis, R.W. Lea, A.J. McIntosh, M.J. Baker, Investigating the rapid diagnosis of gliomas from serum samples using infrared spectroscopy and cytokine and angiogenesis factors, Anal Bioanal Chem, 405 (2013) 7347-7355.

[17] A. Khoshmanesh, M.W. Dixon, S. Kenny, L. Tilley, D. McNaughton, B.R. Wood, Detection and quantification of early-stage malaria parasites in laboratory infected erythrocytes by attenuated total reflectance infrared spectroscopy and multivariate analysis, Anal Chem, 86 (2014) 4379-4386.

[18] Jakobs. BS, Volmer. M, Swinkels. DW, Hofs. MT, Donkervoort. S, Joosting. MM, Wolthers. BG, de Peinder. P, Voorbij. HA, New method for faecal fat determination by mid-infrared spectroscopy, using a transmission cell: animprovement in standardization, Ann Clin Biochem, 37(2000) 343-349.

[19] P. Huang, Y. Ke, Q.Y. Wang, Z.Y. Wang, Analysis of postmortem metabolic changes in rat kidney cortex using Fourier transform infrared spectroscopy, Spectroscopy 22 (2008) 21-31.

[20] Y. Tuo, P. Huang, Y. Ke, S. Fan, Q. Lu, B. Xin, Z. Wang, Attenuated total reflection Fourier transform infrared spectroscopic investigation of the postmortem metabolic process in rat and human kidney cortex, Appl Spectrosc, 64 (2010) 268-274.

[21] Y. Ke, Y. Li, Z.Y. Wang, The changes of fourier transform infrared spectrum in rat brain, J Forensic Sci, 57 (2012) 794-798.

[22] P. Huang, C.P. Su, S.S. Li, B. Xing, Y. Tuo, Q.Y. Lu, Y. Ke, Z.Y. Wang, [Estimation of postmortem interval using FTIR spectroscopy in rats' cardiac muscle], Fa yi xue za zhi, 26 (2010) 1-5.

[23] P. Huang, W.P. Tian, G.D. Yang, Y. Tuo, Z.Y. Wang, [Study on estimation of postmortem interval using FTIR in rat lung], Guang pu xue yu guang pu fen xi = Guang pu, 27 (2007) 1962-1965.

[24] V. Sikirzhytski, K. Virkler, I.K. Lednev, Discriminant analysis of Raman spectra for body fluid identification for forensic purposes, Sensors, 10 (2010) 2869-2884. 

spectroscopy, J Forensic Sci, 56 (2011) 1580-1587.

385 [26] Acquah GE, Via BK, Billor N, Fasina OO, Eckhardt LG, Identifying plant Part Composition of 16(2016) pii:E 1375.

388 [27] M.L. L'igia P. Br' as, Ana P. Ferreira and Jos' e C. Menezes, A bootstrap-based strategy for spectral interval selection in PLS regression, J. Chemometrics, 22 (2008) 659-700.

[28] E. Mistek, I.K. Lednev, Identification of species' blood by attenuated total reflection (ATR) Fourier transform infrared (FT-IR) spectroscopy, Anal Bioanal Chem, 407 (2015) 7435-7442. Vibrational characterization of female gametes: a comparative study, Analyst, 139 (2014) 5049-5060.

394 [30] J. Cao, E.S. Ng, D. McNaughton, E.G. Stanley, A.G. Elefanty, M.J. Tobin, P. Heraud, The characterisation of pluripotent and multipotent stem cells using Fourier transform infrared microspectroscopy, Int J Mol Sci, 14 (2013) 17453-17476.

[31] S. Caine, P. Heraud, M.J. Tobin, D. McNaughton, C.C. Bernard, The application of Fourier transform infrared microspectroscopy for the study of diseased central nervous system tissue, NeuroImage, 59 (2012) 3624-3640.

[32] L.B.M.-g.L. Bachmann, Application of FTIR Spectroscopy for Identification of Blood and 401 Leukemia Biomarkers: A Review over the Past 15 Years, Appl Spectrosc Rev, 46 (2011) 388-404.

403 McNaughton, B.R. Wood, Discriminating the intraerythrocytic lifecycle stages of the malaria parasite using synchrotron FT-IR microspectroscopy and an artificial neural network, Anal Chem, 81 (2009) $2516-2524$.

[34] D. Perez-Guaita, J. Ventura-Gayete, C. Perez-Rambla, M. Sancho-Andreu, S. Garrigues, M. de la Guardia, Protein determination in serum and whole blood by attenuated total reflectance infrared spectroscopy, Anal Bioanal Chem, 404 (2012) 649-656.

[35] S. Pittner, F.C. Monticelli, A. Pfisterer, A. Zissler, A.M. Sanger, W. Stoiber, P. Steinbacher, 410 Postmortem degradation of skeletal muscle proteins: a novel approach to determine the time since 411 death, Int J Legal Med, 130 (2016) 421-431.

412 [36] S. Kumar, W. Ali, S. Bhattacharya, U.S. Singh, A. Kumar, A.K. Verma, The effect of elapsed time 
on cardiac troponin- $\mathrm{T}(\mathrm{cTnT})$ degradation and its relation to postmortem interval in cases of electrocution, J Forensic Leg Med, 34 (2015) 45-49.

[37] S. Kumar, W. Ali, U.S. Singh, A. Kumar, S. Bhattacharya, A.K. Verma, R. Rupani, Temperature-Dependent Postmortem Changes in Human Cardiac Troponin-T (cTnT): An Approach in Estimation of Time Since Death, J Forensic Sci, (2015).

[38] S. Pittner, B. Ehrenfellner, F.C. Monticelli, A. Zissler, A.M. Sanger, W. Stoiber, P. Steinbacher, Postmortem muscle protein degradation in humans as a tool for PMI delimitation, Int J Legal Med, (2016).

[39] G. Clemens, J.R. Hands, K.M. Dorling, M.J. Baker, Vibrational spectroscopic methods for cytology and cellular research, Analyst, 139 (2014) 4411-4444.

[40] M. Mizuguchi, M. Nara, K. Kawano, K. Nitta, FT-IR study of the Ca2+-binding to bovine alpha-lactalbumin. Relationships between the type of coordination and characteristics of the bands due to the Asp COO- groups in the Ca2+-binding site, FEBS Lett, 417 (1997) 153-156.

[41] M. Nara, M. Tanokura, Infrared spectroscopic study of the metal-coordination structures of calcium-binding proteins, Biochem Bioph Res C, 369 (2008) 225-239.

[42] K. Uemura, K. Shintani-Ishida, K. Saka, M. Nakajima, H. Ikegaya, Y. Kikuchi, K. Yoshida, Biochemical blood markers and sampling sites in forensic autopsy, J Forensic Leg Med, 15 (2008) 312-317.

[43] O.V. Allison Derenne, Erik Goormaghtigh, Lipid quantification method using FTIR spectroscopy applied on cancer cell extracts, BBA, 184 (2014) 1200-1209.

[44] T. Williams, S. Soni, J. White, G. Can, G.T. Javan, Evaluation of DNA degradation using flow cytometry: promising tool for postmortem interval determination, Am J Forensic Med Path, 36 (2015) $104-110$

[45] J. Hansen, I. Lesnikova, A.M. Funder, J. Banner, DNA and RNA analysis of blood and muscle from bodies with variable postmortem intervals, Forensic Sci Med Path, 10 (2014) 322-328.

[46] L.A. Johnson, J.A. Ferris, Analysis of postmortem DNA degradation by single-cell gel electrophoresis, Forensic Sci Int, 126 (2002) 43-47.

[47] M. Bauer, I. Gramlich, S. Polzin, D. Patzelt, Quantification of mRNA degradation as possible indicator of postmortem interval--a pilot study, Legal Med, 5 (2003) 220-227.

[48] W.C. Li, K.J. Ma, Y.H. Lv, P. Zhang, H. Pan, H. Zhang, H.J. Wang, D. Ma, L. Chen, Postmortem 
interval determination using 18S-rRNA and microRNA, Sci Justice, 54 (2014) 307-310.

[49] C. Palmiere, P. Mangin, Postmortem chemistry update part I, Int J Legal Med, 126 (2012) 187-198

[50] Bocaz-Beneventi G, Tagliaro F, Bortolotti F, Manetto G, Havel J, Capillary zone electrophoresis and artificial neural networks for estimation of the post-mortem interval (PMI) using electrolytes measurements in human vitreous humour, Int J Legal Med, 116(2002), 5-11.

[51] Kaliszan M, Studies on time of death estimation in the early post mortem period-application of a method based on eyeball temperature measurement to human bodies, Legal Med 15(2013) 278-282.

[52] T. Sato, K. Zaitsu, K. Tsuboi, M. Nomura, M. Kusano, N. Shima, S. Abe, A. Ishii, H Tsuchihashi, K Suzuki, A preliminary study on postmortem interval estimation of suffocated rats by GC-MS/MS-based plasma metabolic profiling, Anal Bioanal Chem, 407(2015) 3659-3665.

[53] RH. Kaszynski, S. Nishiumi, T. Azuma, M. Yoshida, T. Kondo, M. Takahashi, M. Asano, Y. Ueno, Postmortem interval estimation: a novel approach utilizing gas, Anal Bioanal Chem, 408(2016) 3103-3112.

[54] S. Kumar, AK. Verma, Estimation of postmortem interval using the data of insulin level in the cadavers, Data Brief, 2(2016) 354-356.

[55] R. Mukhopadhyay, Portable FTIR spectrometers get moving, Anal Chem, 76 (2004) 369A-372A.

\section{Figure Legends}

Fig 1. FTIR average second-derivative spectra of different PMI groups in the range of 900-1800 and $2800-3100 \mathrm{~cm}^{-1}$.

Fig 2 The results of PCA analysis applied to second derivative spectra of different PMI groups in the $3100-2800$ and $1800-900 \mathrm{~cm}^{-1}$ regions. (A) A two-dimensional score plot along PC1verus PC2. (B) The PC1 loading plot is displayed where the loadings from positive to negative values represent spectral changes with increasing PMI from 0 to $48 \mathrm{~h}$.

Fig 3 The results of cross validation based on calibration samples from 0 to $48 \mathrm{~h}$. (A) A 
474 regression plot between actual and predictive PMI. (B) Corresponding regression loading plot for 475 latent factor 1.

478 Fig 4 The results of cross validation based on calibration samples from 0-24h and 24-48h 479 respectively. (A-B) Regression plots between actual and predictive PMI for the two models. (C-D) 480 Their corresponding regression loading plots for factor 1.

483 Fig 5 The prediction results performed by developed PLS models in a validation dataset. (A)

484 Prediction result for PMI groups before and after 24h postmortem by the PLS model for $0-48 \mathrm{~h}$. 485 The black dot line represents the classification threshold at $24 \mathrm{~h}$. A complete separation between 486 the two postmortem time ranges can be observed. (B) Regression relationship between actual and 487 predictive PMI for external prediction in independent validation sets. This prediction is performed 488 by sub-models for $0-24 \mathrm{~h}$ and $24 \mathrm{~h}-48 \mathrm{~h}$ upon approximate evaluation of model for $0-48 \mathrm{~h}$. 


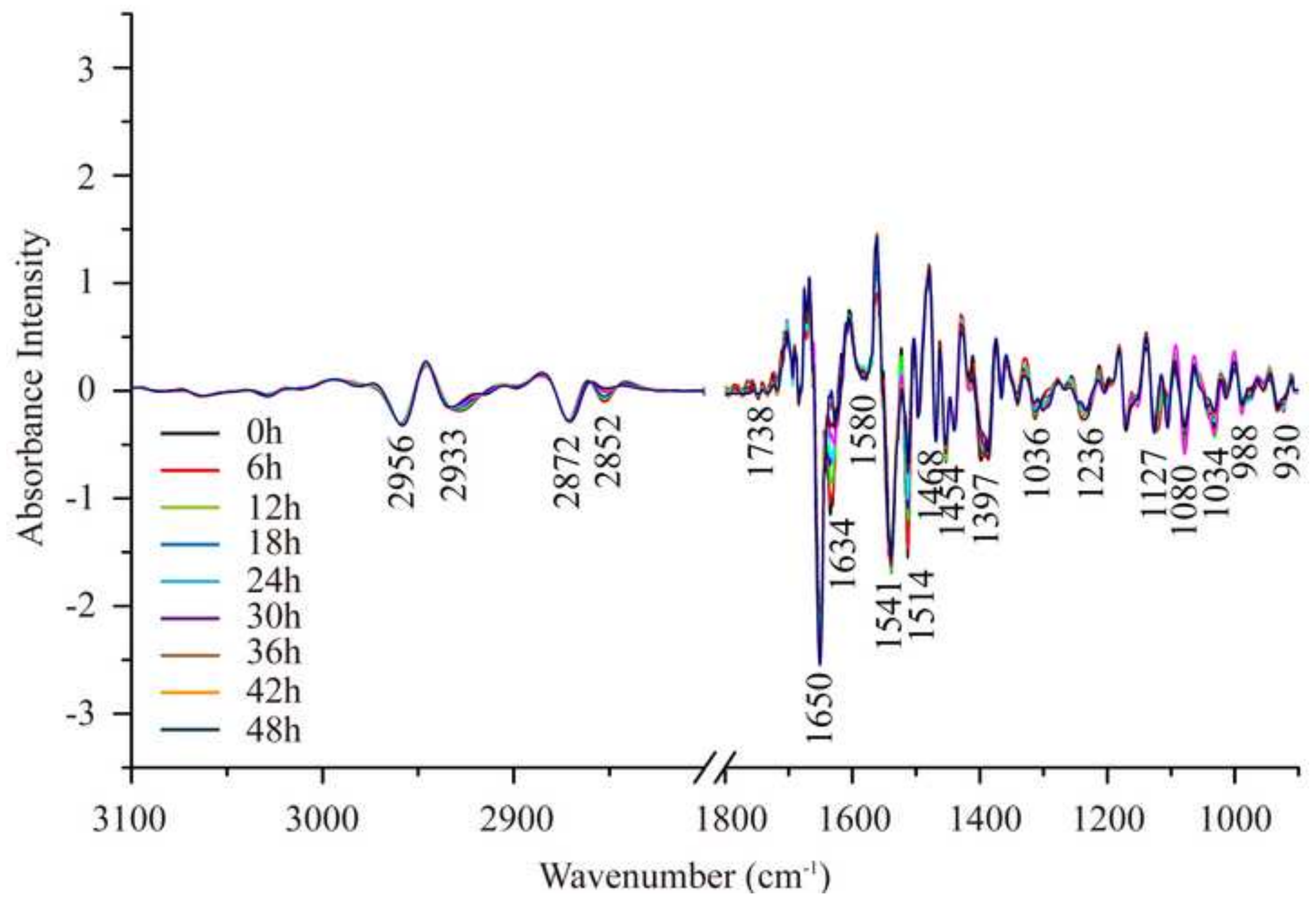


A

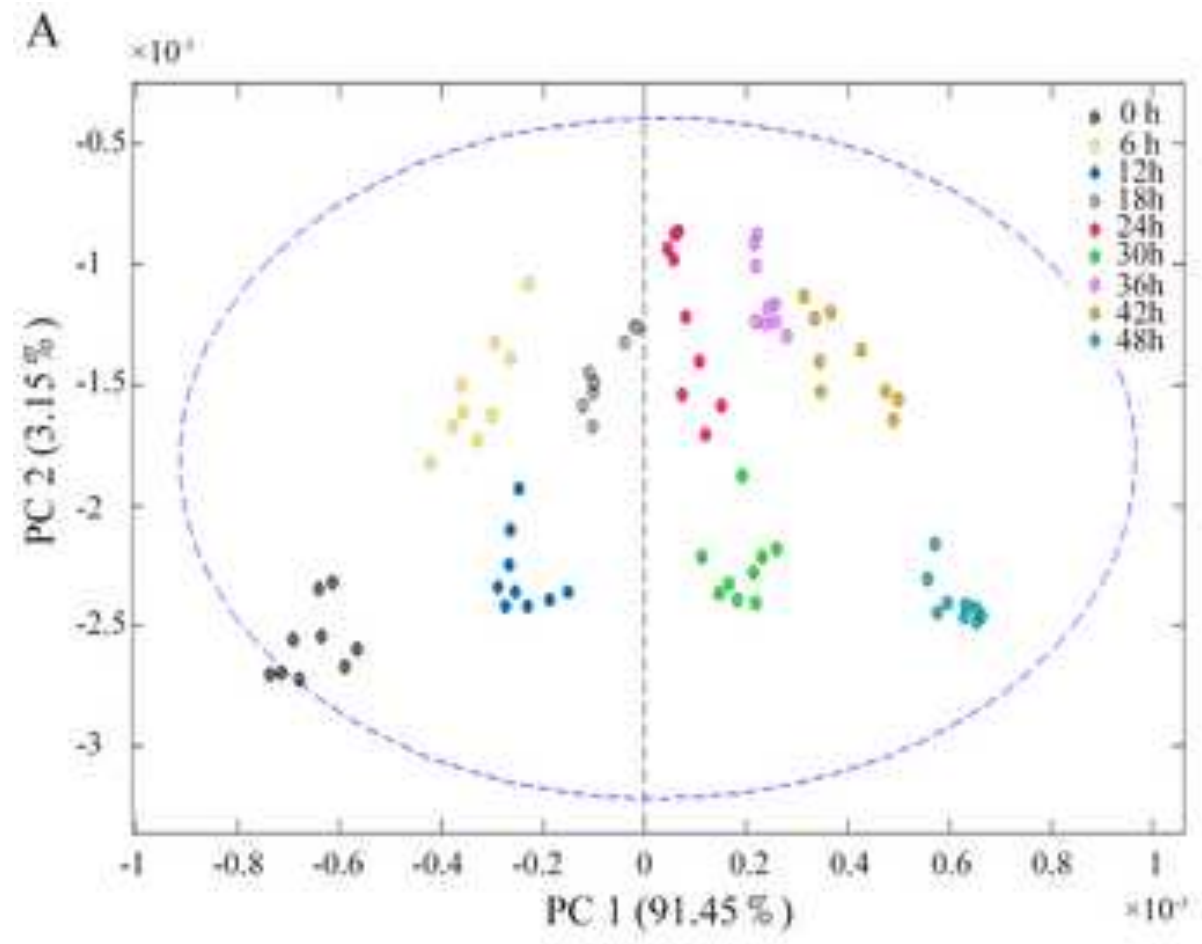

B

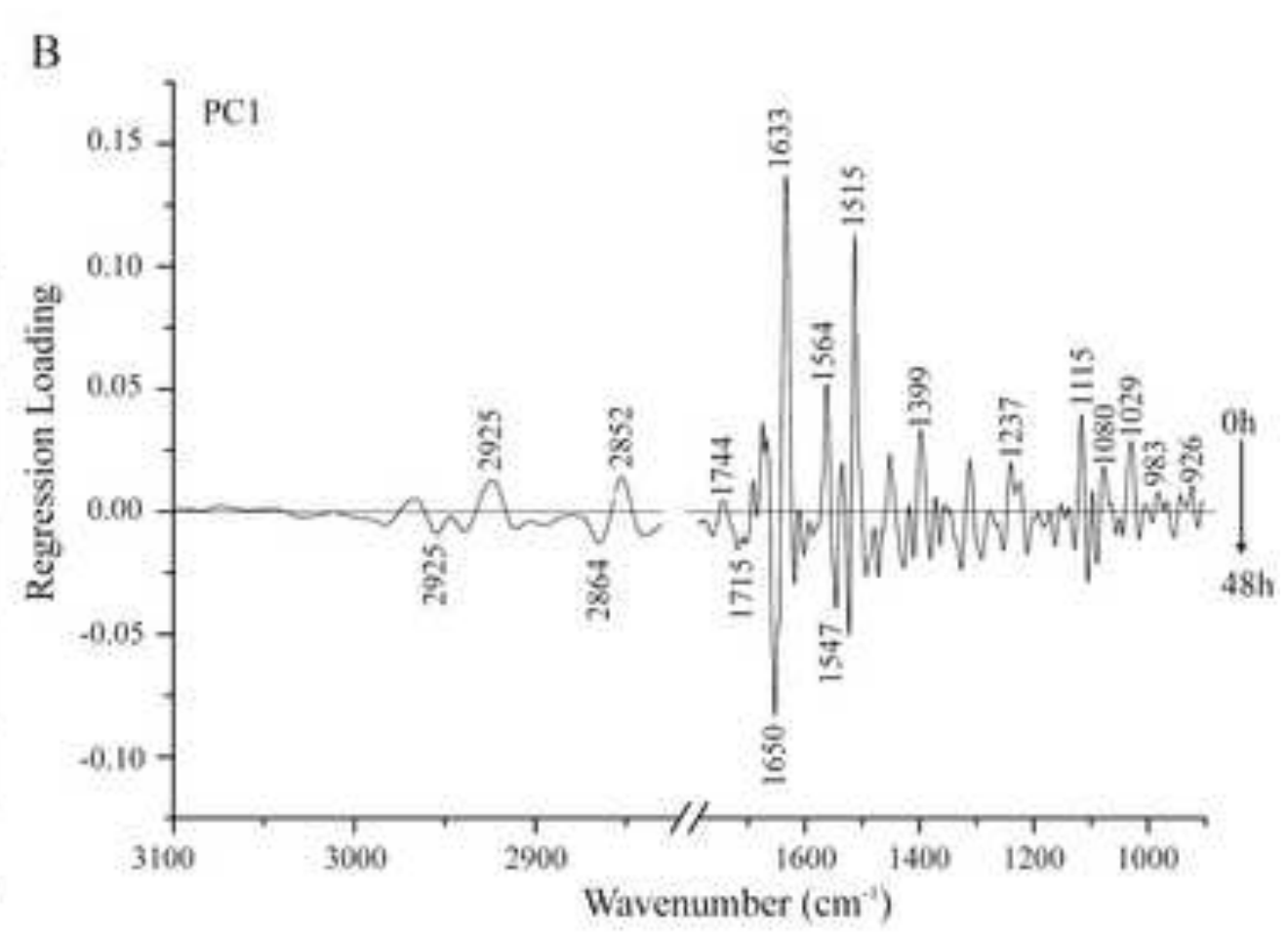


A

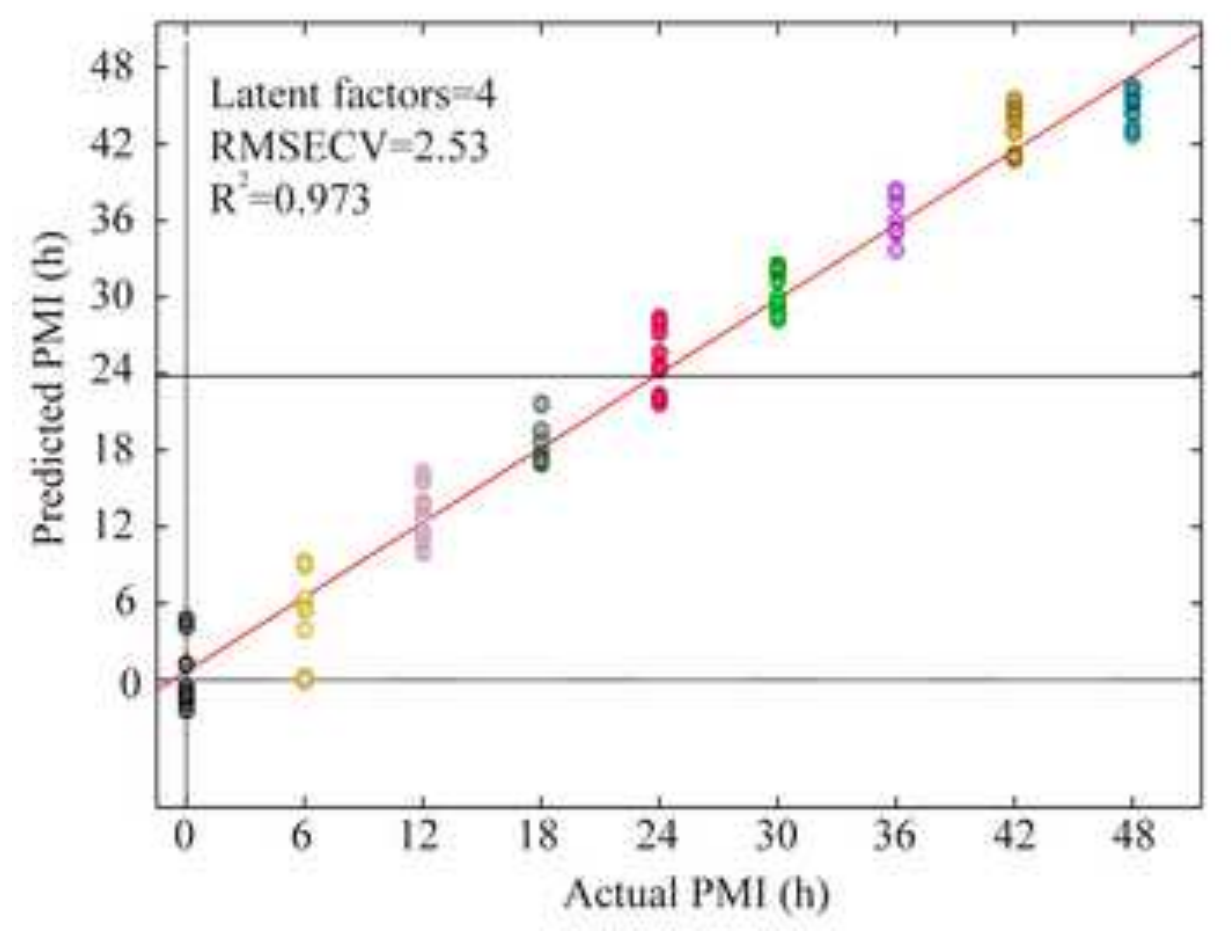

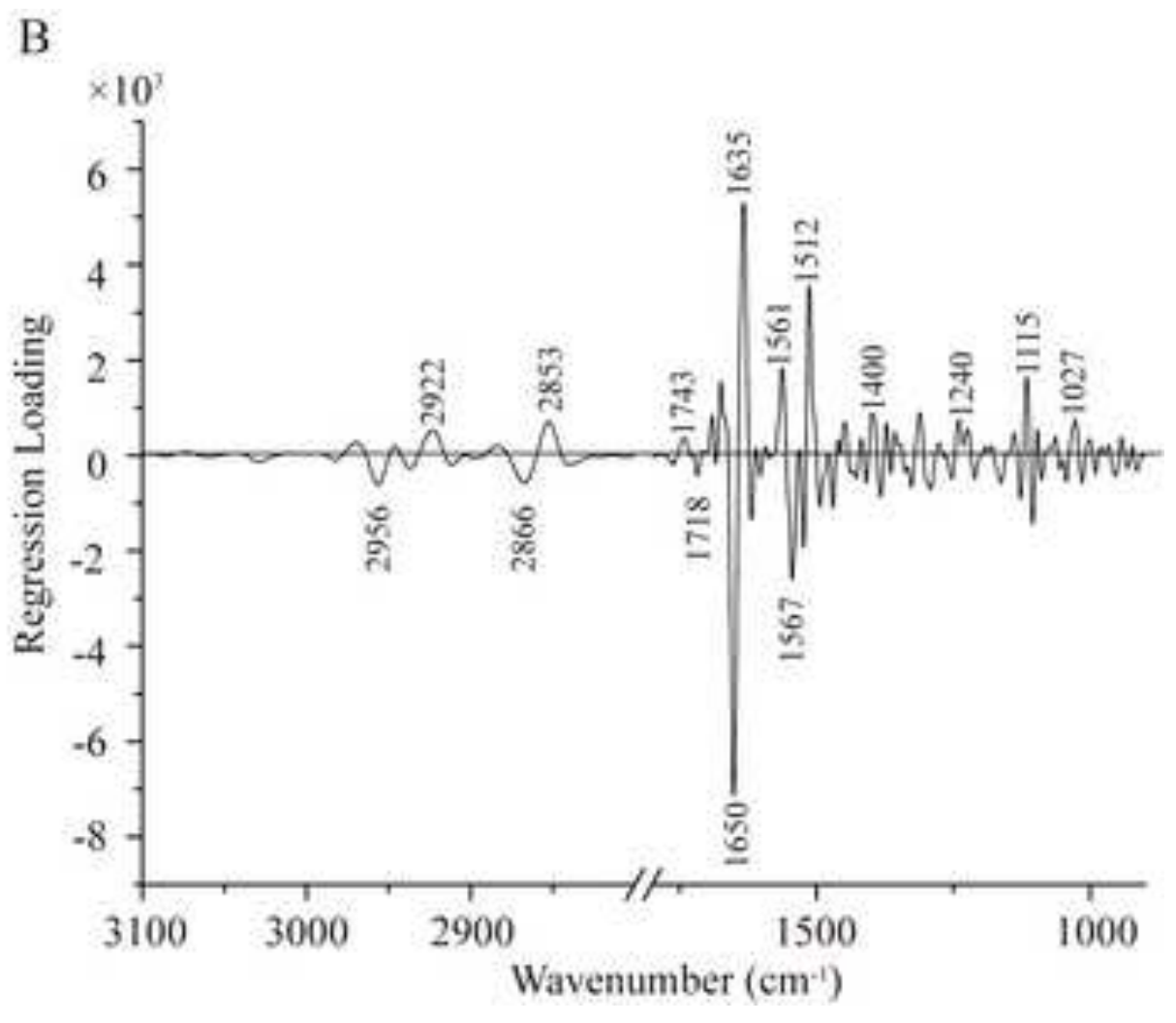


A
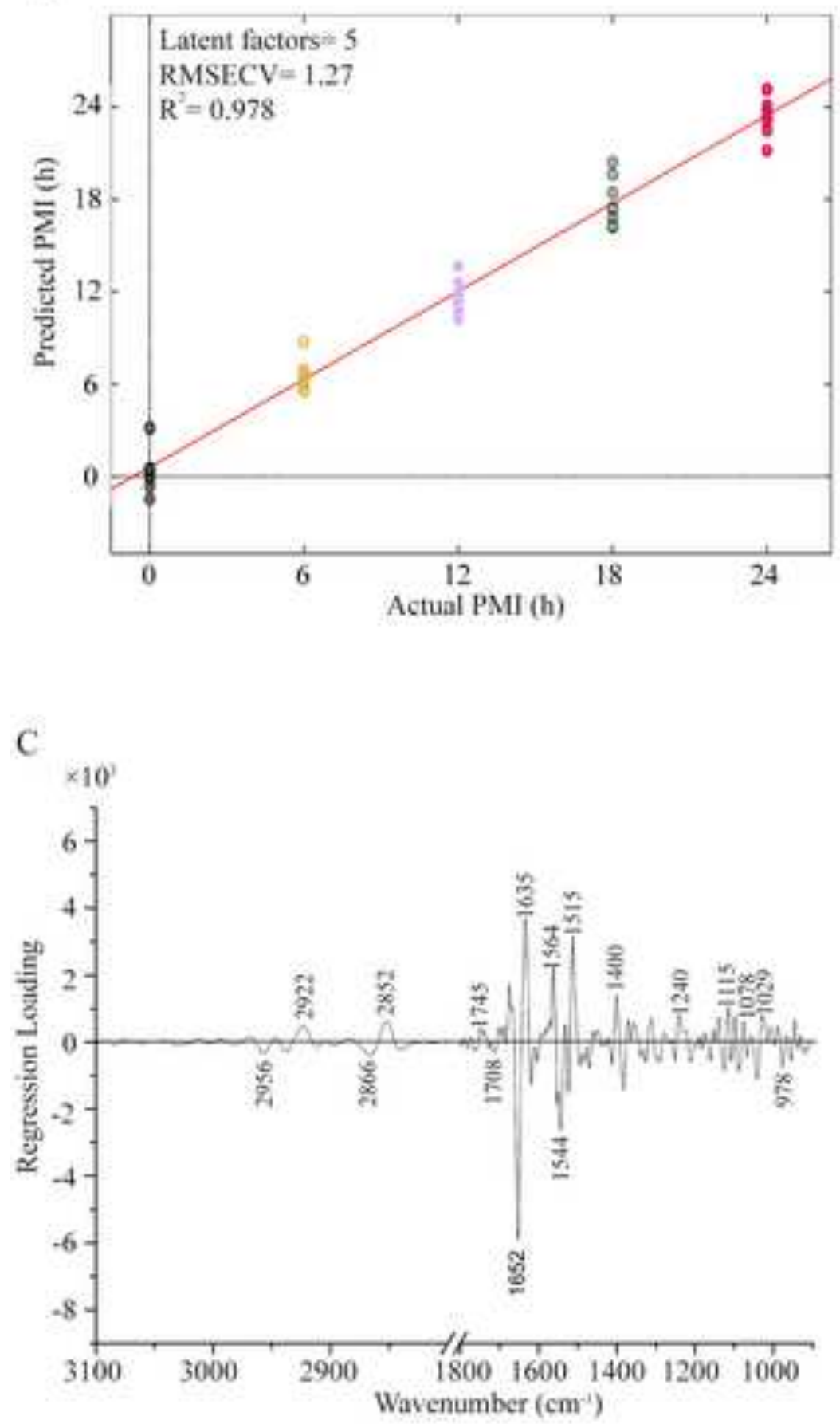

B

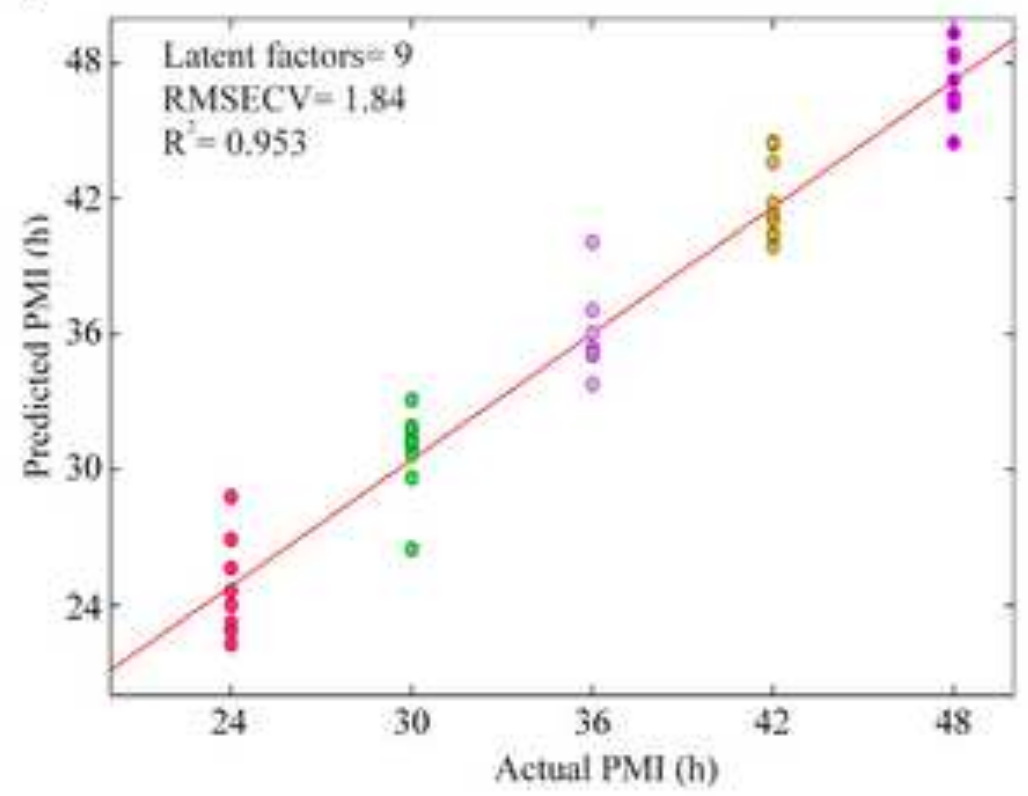

D

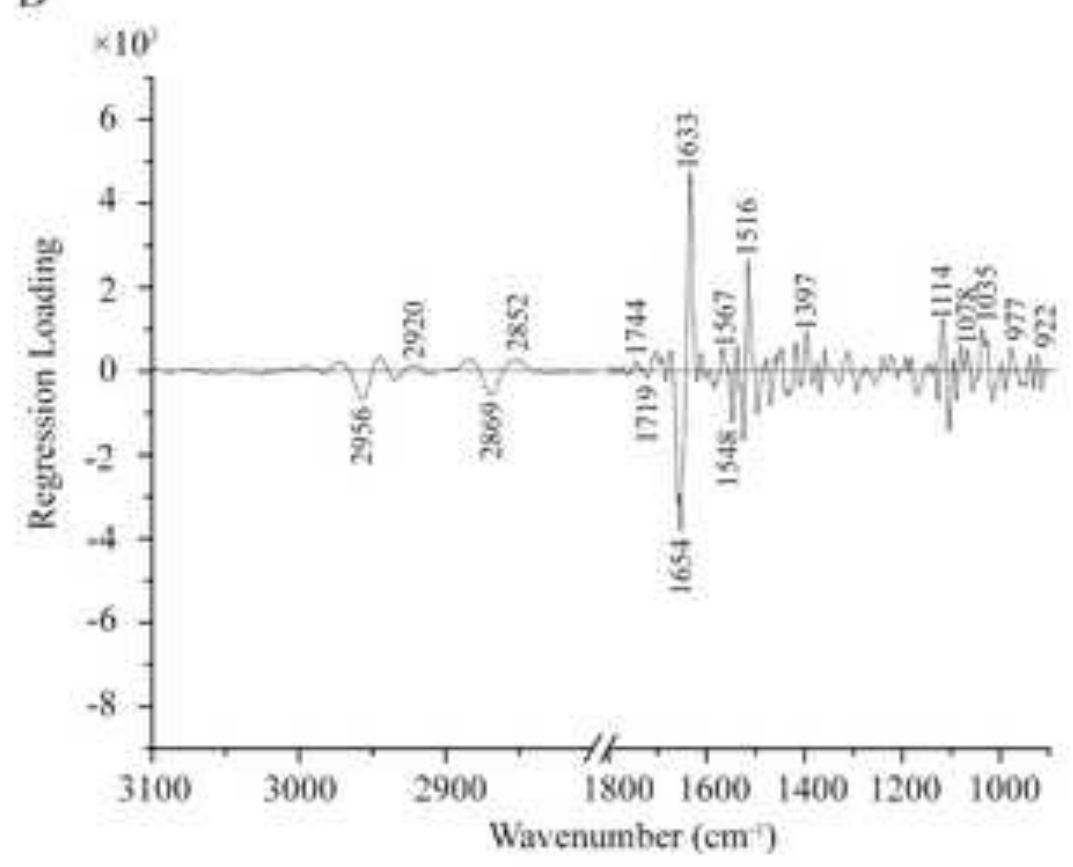


A

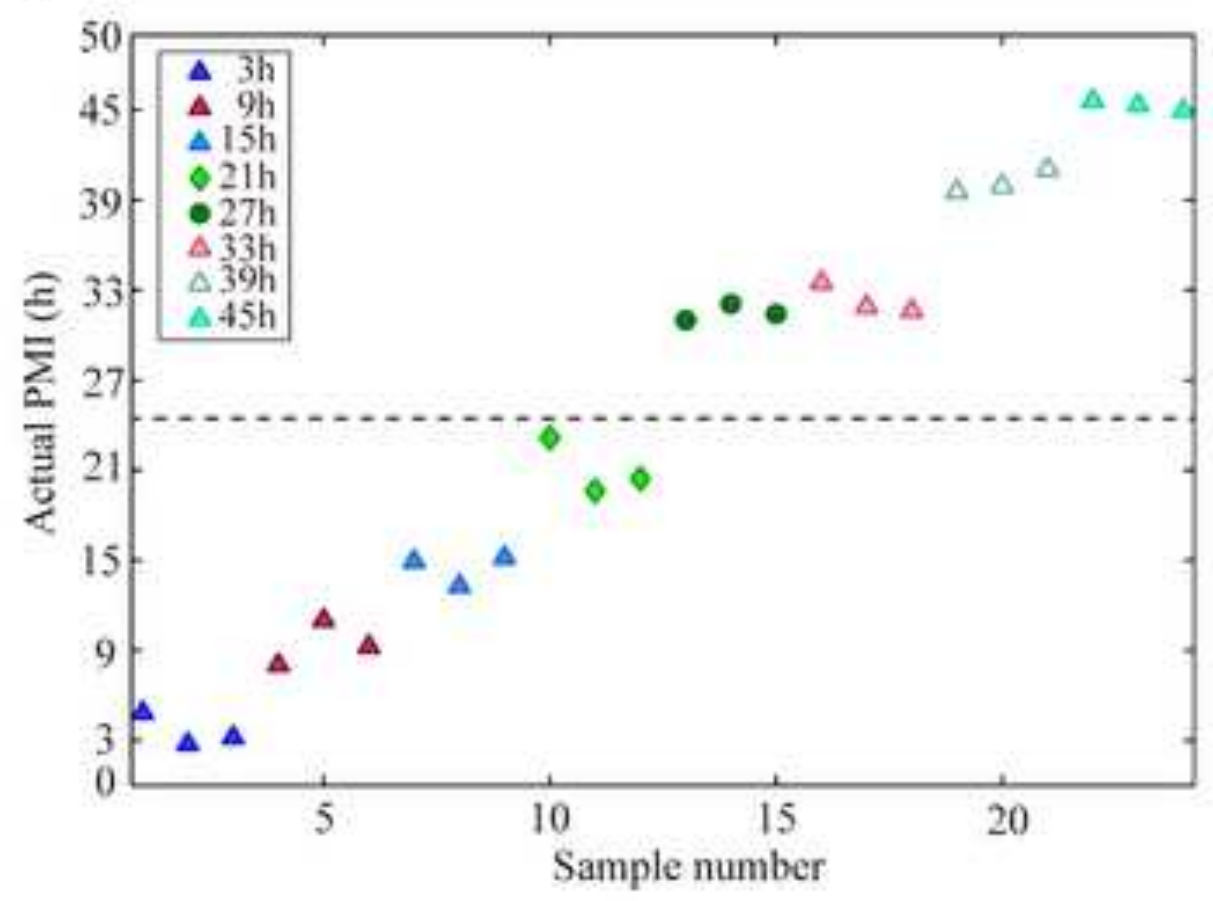

B

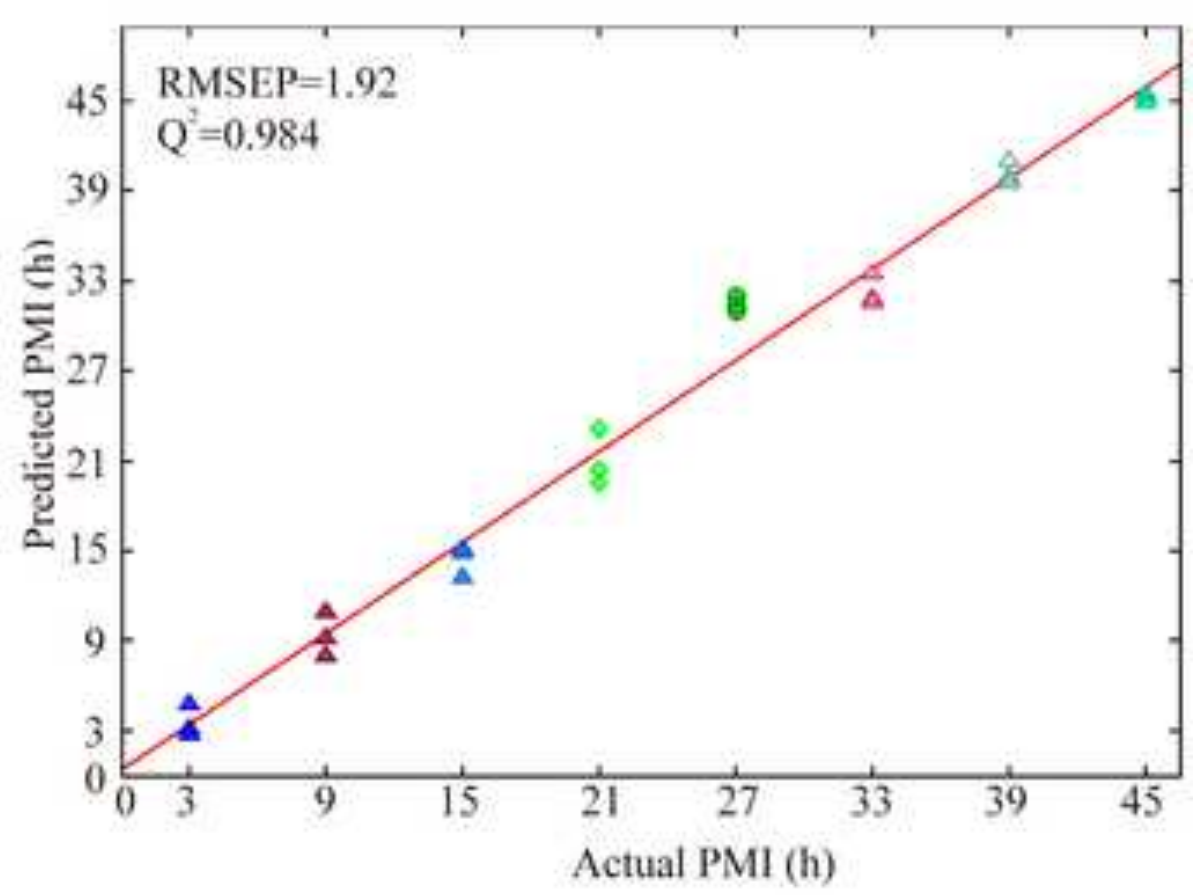


Table 1 Positions of the bands $\left(\mathrm{cm}^{-1}\right)$ with their assignments observed in second derivative FTIR spectra of the plasma studied here

\begin{tabular}{|c|c|}
\hline Frequency $\left(\mathrm{cm}^{-1}\right)$ & Assignment \\
\hline 1300-900 Region & Dominated by phosphate groups and carbohydrates \\
\hline$\sim 925$ & $\mathrm{C}-\mathrm{O}$ and $\mathrm{C}-\mathrm{C}$ vibrations \\
\hline$\sim 988$ & $\mathrm{PO}_{4}^{2-}$ of nucleic acids \\
\hline$\sim 1034$ & C-O stretching vibration \\
\hline$\sim 1080$ & symmetric vibration of $\mathrm{PO}_{2}^{-}$ \\
\hline$\sim 1115$ & C-O vibration of RNA \\
\hline$\sim 1236$ & asymmetric vibration of $\mathrm{PO}_{2}^{-}$ \\
\hline $\begin{array}{l}\text { 1700-1300 Region } \\
\sim 1306\end{array}$ & $\begin{array}{l}\text { Dominated by proteins } \\
\text { amide III band }\end{array}$ \\
\hline$\sim 1397$ & symmetric vibration of $\mathrm{COO}^{-}$ \\
\hline$\sim 1454$ & $\mathrm{C}-\mathrm{H}$ bending from $\mathrm{CH}_{3}$ \\
\hline$\sim 1468$ & $\mathrm{C}-\mathrm{H}$ bending from $\mathrm{CH}_{2}$ \\
\hline$\sim 1514$ & $\mathrm{C}=\mathrm{C}$ vibration of the tyrosine \\
\hline$\sim 1541$ & amide II band \\
\hline$\sim 1584$ & asymmetric vibration of $\mathrm{COO}^{-}$ \\
\hline$\sim 1634$ & $\beta$-pleated sheet structures of protein, Amide I \\
\hline$\sim 1650$ & $\alpha$-helical structures of proteins, Amide I \\
\hline 3050-2800 Region & Dominated by $\mathrm{CH}$ stretches from lipid and proteins \\
\hline$\sim 1738$ & $\mathrm{C}=\mathrm{O}$ stretch of esters \\
\hline$\sim 2852$ & symmetric $\mathrm{C}-\mathrm{H}$ stretch from $\mathrm{CH}_{2}$ \\
\hline$\sim 2872$ & symmetric C-H stretch from $\mathrm{CH}_{3}$ \\
\hline$\sim 2922$ & asymmetric $\mathrm{C}-\mathrm{H}$ stretch from $\mathrm{CH}_{2}$ \\
\hline
\end{tabular}


Table 2 Comparison of the present method with previous methods for PMI estimation

\begin{tabular}{|c|c|c|c|c|c|c|}
\hline Year & Sample & Methods & Parameter & $\begin{array}{c}\text { Max } \\
\text { PMI (h) }\end{array}$ & RMSEP & Ref \\
\hline 2002 & $\begin{array}{c}\text { Human Vitreous } \\
\text { humor }\end{array}$ & $\begin{array}{l}\text { Capillary zone } \\
\text { electrophoresis }\end{array}$ & Electrolyte concentrations & 144 & \pm 3.06 & [50] \\
\hline 2013 & Human eyeball & Thermometer & Temperature & 5.25 & \pm 0.51 & [51] \\
\hline 2015 & Rat plasma & GC/MS & Metabolite concentrations & 48 & \pm 3.41 & [52] \\
\hline 2016 & Mouse muscle & GC/MS & Metabolite concentrations & 48 & \pm 2.65 & [53] \\
\hline \multirow[t]{2}{*}{2016} & human blood & Immunoanalyzer & Insulin concentration & 24 & \pm 3.16 & [54] \\
\hline & Rabbit plasma & FTIR spectroscopy & FTIR spectra & 48 & \pm 1.92 & Present study \\
\hline
\end{tabular}




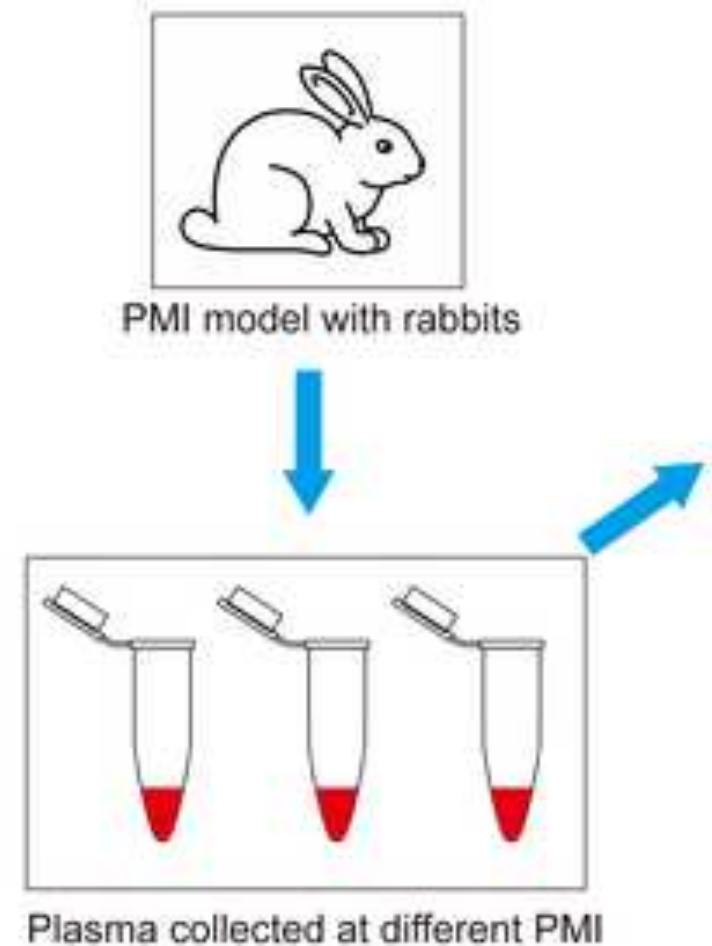

PMI model with rabbits

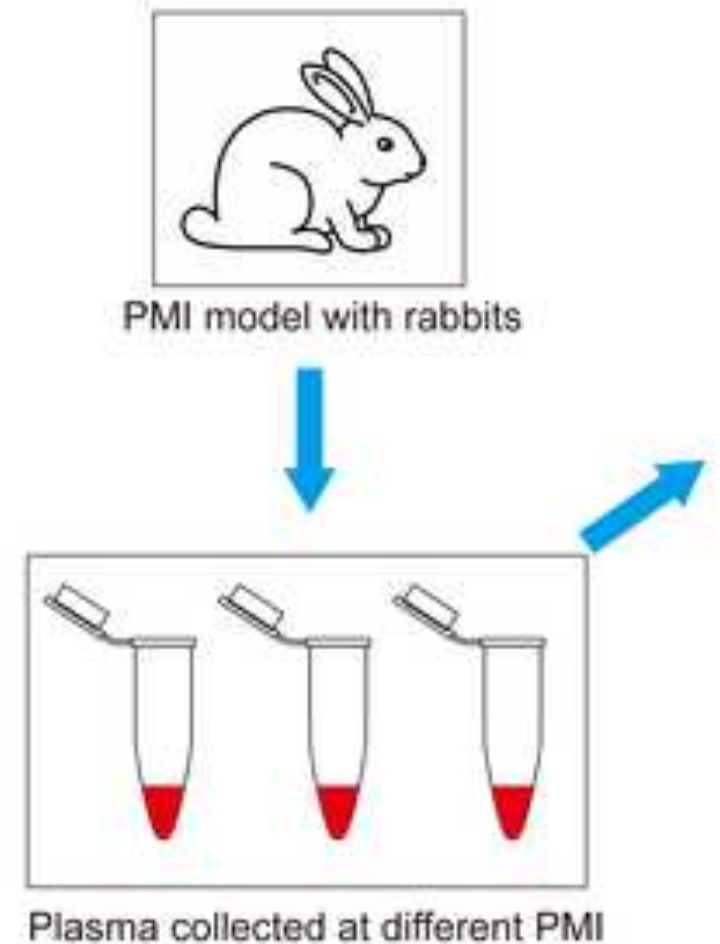

Plasma collected at different PMI

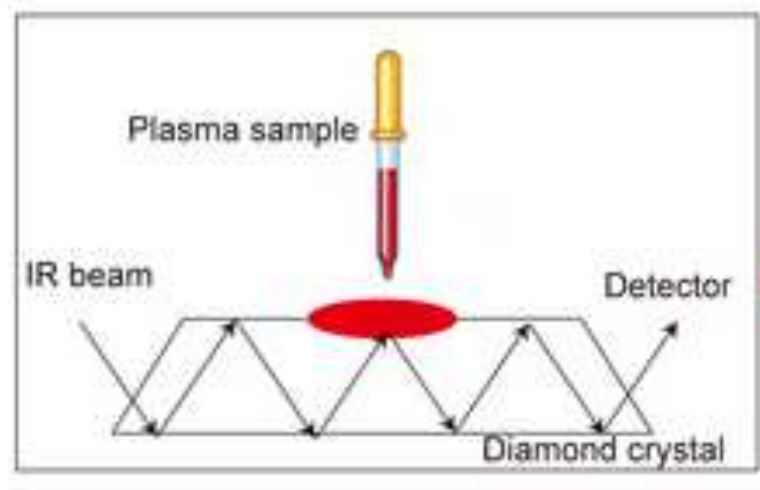

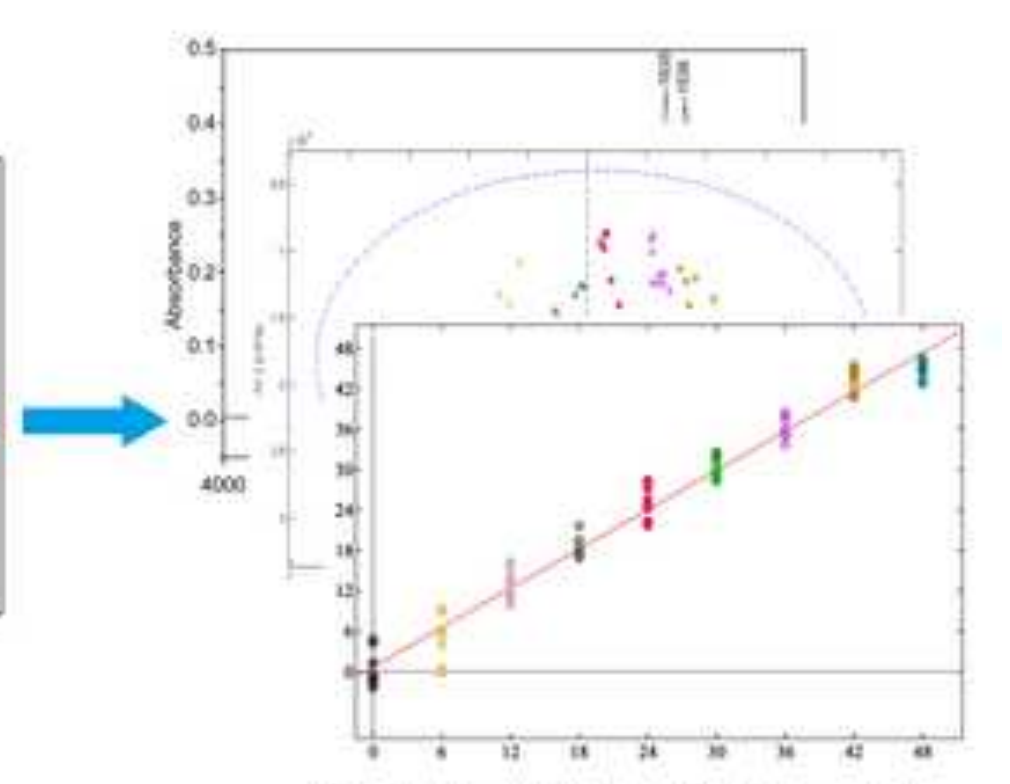

Quality test, PCA and PLS model

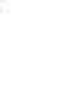

\begin{abstract}
Plasma collected at diferent PMI
\end{abstract}

\author{
$\sqrt{2}$
}

ATR-FTIR measurement

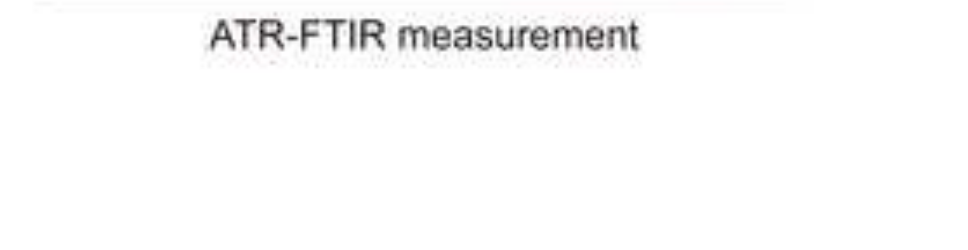

ATR-FTIR measurement 